\title{
Systemic immune-inflammation index is a stage-dependent prognostic factor in patients with operable non-small cell lung cancer
}

\author{
Fangqiu Fu ${ }^{1,2,3,4 \#}$, Chaoqiang Deng ${ }^{1,2,3,4 \#}$, Zhexu Wen ${ }^{1,2,3,4}$, Zhendong Gao ${ }^{1,2,3,4}$, Yue Zhao ${ }^{1,2,3,4}$, \\ Han Han ${ }^{1,2,3,4}$, Shanbo Zheng ${ }^{1,2,3,4}$, Shengping Wang ${ }^{4,5}$, Yuan $\mathrm{Li}^{4,6}$, Hong Hu ${ }^{1,2,3,4}$, Yang Zhang ${ }^{1,2,3,4}$, \\ Haiquan Chen ${ }^{1,2,3,4}$
}

${ }^{1}$ Department of Thoracic Surgery, Fudan University Shanghai Cancer Center, Shanghai, China; ${ }^{2}$ Institute of Thoracic Oncology, Fudan University, Shanghai, China; ${ }^{3}$ State Key Laboratory of Genetic Engineering, School of Life Sciences, Fudan University, Shanghai, China; ${ }^{4}$ Department of Oncology, Shanghai Medical College, Fudan University, Shanghai, China; ${ }^{5}$ Department of Radiology, Fudan University Shanghai Cancer Center, Shanghai, China; ${ }^{6}$ Department of Pathology, Fudan University Shanghai Cancer Center, Shanghai, China

Contributions: (I) Conception and design: F Fu, C Deng, Y Zhang, H Chen; (II) Administrative support: Z Wen, Z Gao, Y Zhao, H Han, S Zheng, S Wang, Y Li, H Hu; (III) Provision of study materials or patients: S Wang, Y Li, H Hu, Y Zhang, H Chen; (IV) Collection and assembly of data: F Fu, C Deng, Z Wen, Y Zhang, H Chen; (V) Data analysis and interpretation: All authors; (VI) Manuscript writing: All authors; (VII) Final approval of manuscript: All authors.

\#These authors contributed equally to this work.

Correspondence to: Haiquan Chen, MD; Yang Zhang, MD. Department of Thoracic Surgery, Fudan University Shanghai Cancer Center, 270 DongAn Road, Shanghai 200032, China. Email: hqchen1@yahoo.com; fduzhangyang1987@hotmail.com.

Background: Immune function is a key component affecting tumor progression in patients with cancer. The purpose of this study was to identify the prognostic value of systemic immune-inflammation index (SII) in patients with non-small cell lung cancer (NSCLC) and the differences of its prognostic value in patients with distinct characteristics.

Methods: Patients with completely resected NSCLC were reviewed according to the eighth TNM classification of lung cancer. Patients were further categorized into the low- and high-SII groups. Cox proportional hazard analyses were performed to identify the independent prognostic factors.

Results: A total of 3984 patients with NSCLC were enrolled in this study. Kaplan-Meier analyses demonstrated that high SII was associated with worse recurrence-free survival $(\mathrm{RFS})(\mathrm{P}<0.001)$ and overall survival (OS) $(\mathrm{P}<0.001)$. Cox proportional hazard analyses revealed that SII was an independent risk factor for worse RFS $(\mathrm{P}=0.038)$ and $\mathrm{OS}(\mathrm{P}=0.043)$. Further analyses demonstrated that the prognostic value of SII was observed only in patients with stage I disease $(\mathrm{P}<0.001)$, solid nodules $(\mathrm{P}=0.002)$, or adenocarcinoma $(\mathrm{P}<0.001)$. Sensitivity analyses using multiple imputation and competing risk analyses also confirmed similar results.

Conclusions: SII was associated with worse survival independently, and its prognostic role was exhibited solely in NSCLC patients with stage I disease, solid nodules, and adenocarcinoma. This study helped us specify the target population for clinical use of SII.

Keywords: Non-small cell lung cancer (NSCLC); systemic immune-inflammation index (SII); prognosis

Submitted Apr 06, 2021. Accepted for publication Jun 18, 2021.

doi: $10.21037 /$ tlcr-21-267

View this article at: https://dx.doi.org/10.21037/tlcr-21-267 


\section{Introduction}

The immune system helps clear abnormal cells and pathogens in the body (1). Systemic inflammation has emerged as a significant part in the development and progression of cancer $(2,3)$. Besides the individual characteristics of tumor, host systemic immune-inflammatory response also contributed to tumorigenesis (4). Prognostic factors, which were easily accessible and cost-effective, have been investigated to evaluate the risk of recurrence or death after curative-intent surgery. Systemic immune-inflammation index (SII), based on the blood routine examination results, was reported to be a useful prognostic indicator in some solid tumors, including small and non-small cell lung cancer (NSCLC) (5-8). However, no data exist revealing the prognostic value of SII in NSCLC patients with distinct TNM stage, CT appearance, and pathology.

In the present study, in a large scale, we investigated the prognostic role of SII in patients with NSCLC undergoing curative-intent surgery. More importantly, we compared prognostic roles of SII in patients with different stage, CT appearance, and pathology. We presented the following article in accordance with the REMARK reporting checklist (available at https://dx.doi.org/10.21037/tlcr-21-267).

\section{Methods}

\section{Patients}

The retrospective study was carried out in consecutive patients who underwent upfront curative-intent resection of pathologic stage I-IIIA NSCLC between April 2008 and December 2015 at the Department of Thoracic Surgery at Fudan Shanghai Cancer Center (FUSCC). Patients receiving neoadjuvant therapy were excluded. Patients with no follow-up data, no results of blood routine examination, or missing images of high-resolution computed tomography (HRCT) were excluded. The work flow of this study was showed in Figure S1. The following variables were prospectively collected: age, sex, smoking history, the radiologic appearance in HRCT, results of blood routine examination, operative procedures, pathologic size, pathologic stage, histology, lymphovascular invasion (LVI), visceral pleural invasion (VPI), adjuvant chemotherapy, adjuvant radiotherapy, and data of followup. The follow-up protocol for surgically resected NSCLC patients was described before (9). The study was conducted in accordance with the Declaration of Helsinki (as revised in 2013). The study was approved by Institutional Review
Board of FUSCC (IRB\#090977-1) and individual consent for this retrospective analysis was waived.

The whole blood was drawn within 5 days before surgery, and concentrations of platelets, neutrophils, and lymphocytes were measured in the central laboratory of FUSCC. The last results of routine blood examinations before surgery was used to calculate the SII for each patient. SII was calculated as follows: SII $=$ platelet $\times$ neutrophil $/$ lymphocyte.

\section{Radiologic and histologic evaluation}

Images of HRCT were reviewed by two radiologists independently for radiologic appearance. Groundglass opacity (GGO) was defined as a radiologic finding showing a hazy opacity with the presence of the underlying pulmonary vessels or bronchial structures (10). Lung nodules only consisting of GGO were categorized as pure GGOs, while those only consisting of solid part as solid nodules. The rest nodules containing GGO and solid parts simultaneously were categorized as mixed GGOs. The interobserver variability was described in our previous study $(9,11)$. GGO components were considered to be present in pure GGOs and mixed GGOs.

Adenocarcinoma subtypes were classified according to the lung adenocarcinoma classification of WHO (12). Lung adenocarcinoma was classified into adenocarcinoma in situ (AIS), minimally invasive adenocarcinoma (MIA), lepidic pattern-predominant adenocarcinoma (LPA), acinar pattern-predominant adenocarcinoma (APA), papillary pattern-predominant adenocarcinoma (PPA), invasive mucinous adenocarcinoma (IMA), micropapillary patternpredominant adenocarcinoma (MPA) and solid patternpredominant adenocarcinoma (SPA). Pathological size was defined as the maximum diameter on the maximum dimension of specimens. LVI was defined as the presence of cancer cells in the lymphatic and vascular vessels, and VPI was defined as the presence of cancer cells within the visceral pleura. The TNM stage was evaluated according to the eighth TNM classification of lung cancer (13). The findings were reviewed by two experienced pathologists, and disagreements were resolved through discussion.

\section{Statistical analysis}

Overall survival (OS) was considered to be the time between surgery and death or last follow-up. Recurrencefree survival (RFS) was defined as the time from surgery to 
first recurrence or last follow-up. Patients who died from other causes were considered to be censored with no event when calculating RFS. The median follow-up time was 45.1 months. Differences of characteristics between patients with high SII and low SII were evaluated by two-sample $t$-tests for continuous variables and by Chi-square test or Fisher exact tests for categorical variables, respectively. The patients were grouped into high- and low-SII by optimal cut-off using function surv_cutpoint (R package survminer). High SII was defined as SII $>479$, while low $\mathrm{SII}$ as $\mathrm{SII} \leq 479$. Missing data of GGO appearance and SII were filled by multiple imputation for sensitivity analyses. To eliminate the effect of non-cancer-related death on the main results, competing risk analyses using $\mathrm{R}$ package "cmprsk" were conducted.

Data were analyzed by SPSS software (version 25.0, IBM Corp, Armonk, NY, USA) and R software (version 3.6.0). Univariate Cox proportional hazards models were used to obtain potential risk factors for RFS and OS. The independent prognostic roles of variables were assessed by multivariate Cox proportional hazards models. All tests were two-tailed, and the statistical significance was set at $\mathrm{P}<0.05$.

\section{Results}

\section{Clinicopathological characteristics}

A total of 3,984 patients with resectable NSCLC were enrolled in this study (Figure S1). There were 637 (16.0\%) patients with pure GGO, 961 (24.1\%) patients with mixed GGO, and 2,386 (59.9\%) patients with solid nodules. Among these patients, 2,682 (67.3\%) patients had stage 0/I diseases, while 1,302 (32.7\%) patients had stage II/ III diseases. The optimal cut-off for SII, determined by surv_cutpoint (R package survminer), was 479 . There were 1,643 patients in the high-SII group and 2,341 patients in the low-SII group. The level of SII was significantly associated with age $(\mathrm{P}=0.011)$, sex $(\mathrm{P}<0.001)$, smoking history $(\mathrm{P}<0.001)$, CT appearance $(\mathrm{P}<0.001)$, operative procedure $(\mathrm{P}<0.001)$, pathologic size $(\mathrm{P}<0.001)$, p-TNM stage $(\mathrm{P}<0.001)$, histology $(\mathrm{P}<0.001)$, LVI $(\mathrm{P}<0.001)$, VPI $(\mathrm{P}<0.001)$, adjuvant chemotherapy $(\mathrm{P}<0.001)$, and adjuvant radiotherapy $(\mathrm{P}=0.001)$. Clinicopathological characteristics were shown in Table 1.

The median follow-up time was 45.1 months. The 5-year RFS was $69.9 \%$, and the 5 -year OS was $84.5 \%$. During follow-up, 956 patients developed disease recurrence and
484 patients died.

\section{The effect of SII on RFS}

In order to investigate the prognostic value of SII, survival analyses stratified by SII were performed. The results demonstrated that high SII was associated with worse RFS $(\mathrm{P}<0.0001$, Figure 1A). Since non-cancer-related death was a competing risk for lung cancer recurrence, competing risk analyses were also conducted. The results also revealed that high SII was correlated with worse RFS (Figure S2).

Cox proportional hazard models were further used to identify independent factors associated with RFS. The following variables were associated with poor RFS in univariate Cox proportional hazard regression (Table 2): age $(\mathrm{P}<0.001)$, sex $(\mathrm{P}<0.001)$, smoking history $(\mathrm{P}<0.001)$, GGO components $(\mathrm{P}<0.001)$, SII $(\mathrm{P}<0.001)$, p-TNM stage $(\mathrm{P}<0.001)$, histology $(\mathrm{P}<0.001)$, LVI $(\mathrm{P}<0.001)$, VPI $(\mathrm{P}<0.001)$, adjuvant chemotherapy $(\mathrm{P}<0.001)$, and adjuvant radiotherapy $(\mathrm{P}<0.001)$. The multivariate analyses demonstrated age [hazard ratio (HR): 1.009, 95\% confidence interval (CI): 1.002-1.016, $\mathrm{P}=0.011$ ], sex (HR: 1.237, 95\% CI: $1.032-1.482, \mathrm{P}=0.021)$, GGO components (HR: 3.504, 95\% CI: 2.729-4.500, $\mathrm{P}<0.001$ ), SII (HR: 1.154, 95\% CI: 1.011-1.317, $\mathrm{P}=0.034), \mathrm{p}-\mathrm{TNM}$ stage (0/I, reference; II, HR: 2.137, 95\% CI: 1.718-2.658, $\mathrm{P}<0.001$; III, HR: 4.447, 95\% CI: 3.623-5.458, $\mathrm{P}<0.001$; overall $\mathrm{P}<0.001$ ), histology [non-MPA/SPA adenocarcinoma, reference; MPA/SPA, HR: 1.232, 95\% CI: 1.024-1.481, $\mathrm{P}=0.027$; squamous cell carcinoma (SQCC), HR: 0.736, 95\% CI: 0.612-0.883, $\mathrm{P}=0.001$; others, HR: $1.228,95 \% \mathrm{CI}$ : 0.970-1.555, $\mathrm{P}=0.088$; overall $\mathrm{P}<0.001]$, LVI (HR: 1.254 , 95\% CI: 1.071-1.468, $\mathrm{P}=0.005)$, VPI (HR: $1.478,95 \%$ CI: 1.281-1.704, $\mathrm{P}<0.001$ ), and adjuvant radiotherapy (HR: 1.583 , 95\% CI: $1.280-1.959, \mathrm{P}<0.001)$ were able to predict RFS independently in patients with NSCLC (Table 2). Therefore, SII was an independent predictor for RFS in patients with stage I-IIIA NSCLC.

To investigate the effect of missing value of GGO appearance and SII, multiple imputation was used to fill the missing data. Based on the Cox proportional hazard regression of 4,220 patients, the results were similar, and SII still independently predicted survival outcomes in patients with NSCLC (Table S1).

\section{The effect of SII on OS}

Besides RFS, we also investigated the effect of SII on OS. 
Table 1 Clinicopathological characteristics of enrolled patients stratified by SII

\begin{tabular}{|c|c|c|c|c|}
\hline Variables & All patients $(n=3,984)$ & Low SII (SII $\leq 479, n=2,341)$ & High SII (SII >479, n=1,643) & $P$ values \\
\hline Median [IQR] & $60[53,66]$ & $60[53,66]$ & $60[54,67]$ & \\
\hline Mean \pm SD & $59.1 \pm 10.2$ & $58.8 \pm 10.3$ & $59.6 \pm 10.0$ & \\
\hline Sex, n (\%) & & & & $<0.001$ \\
\hline Male & $2,139(53.7)$ & $1,109(47.4)$ & $1,030(62.7)$ & \\
\hline Smoking history, n (\%) & & & & $<0.001$ \\
\hline Never & $2,411(60.5)$ & $1,582(67.6)$ & $819(50.5)$ & \\
\hline Ever & $1,573(39.5)$ & $759(32.4)$ & $814(49.5)$ & \\
\hline Mixed GGO & $961(24.1)$ & $671(28.7)$ & $290(17.7)$ & \\
\hline Solid nodules & $2,386(59.9)$ & $1,208(51.6)$ & $1,178(71.7)$ & \\
\hline Operative procedure, n (\%) & & & & $<0.001$ \\
\hline Wedge resection & $503(12.6)$ & 359 (15.3) & $144(8.8)$ & \\
\hline Segmentectomy & $164(4.1)$ & $118(5.0)$ & $46(2.8)$ & \\
\hline Lobectomy & $2,870(72.0)$ & $1,683(71.9)$ & $1,187(72.2)$ & \\
\hline Bi-lobectomy & $191(4.8)$ & $86(3.7)$ & $105(6.4)$ & \\
\hline Sleeve resection & $102(2.6)$ & $43(1.8)$ & $59(3.6)$ & \\
\hline $\mathrm{IA}$ & $1,825(45.9)$ & $1,253(53.5)$ & $572(34.8)$ & \\
\hline IB & $645(16.2)$ & $349(14.9)$ & $296(18.0)$ & \\
\hline II & $563(14.1)$ & $225(9.6)$ & 338 (20.6) & \\
\hline III & 739 (18.5) & 358 (15.3) & 381 (23.2) & \\
\hline Histology, n (\%) & & & & $<0.001$ \\
\hline AIS & $212(5.3)$ & $156(6.7)$ & $56(3.4)$ & \\
\hline MIA & 467 (11.7) & 335 (14.3) & $132(8.0)$ & \\
\hline LPA & 264 (6.6) & 187 (8.0) & $77(4.7)$ & \\
\hline APA & 1,297 (32.6) & $830(35.5)$ & 467 (28.4) & \\
\hline PPA & $264(6.6)$ & $172(7.3)$ & $92(5.6)$ & \\
\hline IMA & $97(2.4)$ & $69(2.9)$ & $28(1.7)$ & \\
\hline
\end{tabular}

Table 1 (continued) 
Table 1 (continued)

\begin{tabular}{|c|c|c|c|c|}
\hline Variables & All patients $(n=3,984)$ & Low SII (SII $\leq 479, n=2,341)$ & High SII (SII >479, n=1,643) & $P$ values \\
\hline MPA & $39(1.0)$ & $21(0.9)$ & $18(1.1)$ & \\
\hline SPA & $322(8.1)$ & $158(6.7)$ & $164(10.0)$ & \\
\hline SQCC & $784(19.7)$ & $313(13.4)$ & $471(28.7)$ & \\
\hline LVI, n (\%) & & & & $<0.001$ \\
\hline Yes & $461(11.6)$ & $233(10.0)$ & $228(13.9)$ & \\
\hline No & $3,523(88.4)$ & $2,108(90.0)$ & $1,415(86.1)$ & \\
\hline VPI, n (\%) & & & & 0.009 \\
\hline Adjuvant chemotherapy, n (\%) & & & & $<0.001$ \\
\hline Yes & $1,294(32.5)$ & $599(25.6)$ & $695(42.3)$ & \\
\hline No & $2,690(67.5)$ & $1,742(74.4)$ & $948(57.7)$ & \\
\hline Adjuvant radiotherapy, n (\%) & & & & 0.001 \\
\hline Yes & $156(3.9)$ & $71(3.0)$ & $85(5.2)$ & \\
\hline No & $3,828(96.1)$ & $2,270(97.0)$ & $1,558(94.8)$ & \\
\hline
\end{tabular}

SII, systemic immune-inflammation index; IQR, interquartile range; SD, standard deviation; CT, computed tomography; GGO, groundglass opacity; AIS, adenocarcinoma in situ; MIA, minimally invasive adenocarcinoma; LPA, lepidic pattern-predominant adenocarcinoma; APA, acinar pattern-predominant adenocarcinoma; PPA, papillary pattern-predominant adenocarcinoma; IMA, invasive mucinous adenocarcinoma; MPA, micropapillary pattern-predominant adenocarcinoma; SPA, solid pattern-predominant adenocarcinoma; SQCC, squamous cell carcinoma; LVI, lymphovascular invasion; VPI, visceral pleural invasion.
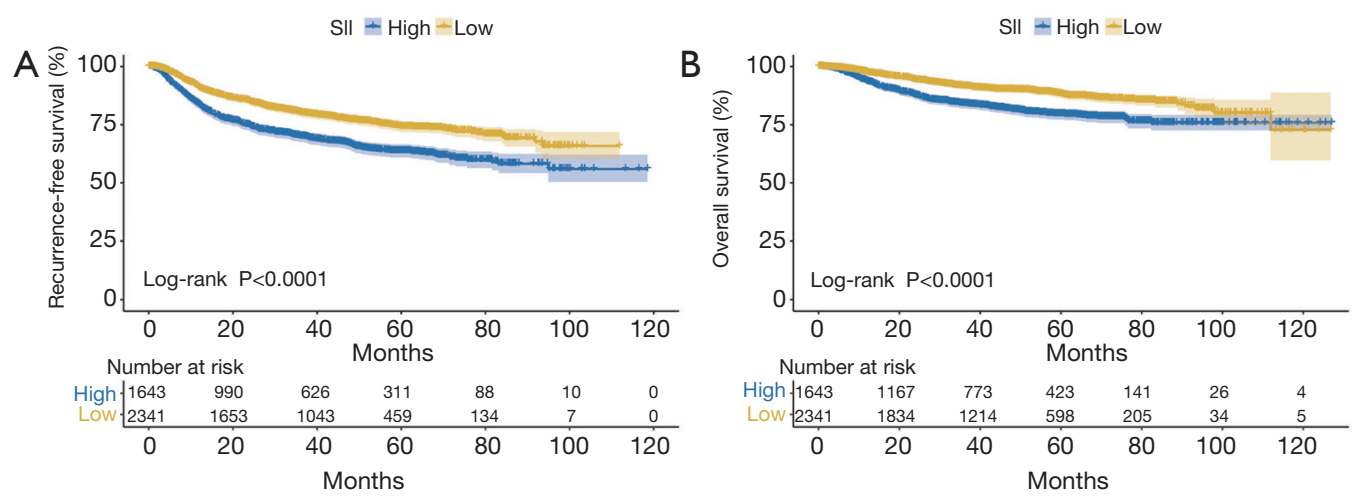

Figure 1 Kaplan-Meier curves of RFS (A) and OS (B) according to the level of SII in patients with NSCLC. High SII was defined as more than 479, while low SII as no more than 479. RFS, recurrence-free survival; OS, overall survival; SII, systemic immune-inflammation index; NSCLC, non-small cell lung cancer. 
Table 2 Univariate and multivariable Cox regression analysis of factors associated with RFS $(\mathrm{n}=3,984)$

\begin{tabular}{|c|c|c|c|c|}
\hline Variables & \multicolumn{2}{|c|}{ Univariate } & \multicolumn{2}{|c|}{ Multivariate } \\
\hline Age & $1.015(1.008,1.022)$ & $<0.001$ & $1.009(1.002,1.016)$ & 0.011 \\
\hline Sex (male vs. female) & $1.844(1.612,2.109)$ & $<0.001$ & $1.237(1.032,1.482)$ & 0.021 \\
\hline Smoking history & $1.672(1.473,1.899)$ & $<0.001$ & $1.085(0.913,1.290)$ & 0.352 \\
\hline SII (high vs. low) & $1.630(1.435,1.850)$ & $<0.001$ & $1.154(1.011,1.317)$ & 0.034 \\
\hline p-TNM stage & & $<0.001$ & & $<0.001$ \\
\hline $0 / l$ & Reference & & Reference & \\
\hline II & $3.645(3.045,4.363)$ & $<0.001$ & $2.137(1.718,2.658)$ & $<0.001$ \\
\hline Non-MPA/SPA adenocarcinoma & Reference & & Reference & \\
\hline MPA/SPA & $3.246(2.720,3.875)$ & $<0.001$ & $1.232(1.024,1.481)$ & 0.027 \\
\hline SQCC & $1.844(1.575,2.160)$ & $<0.001$ & $0.736(0.612,0.883)$ & 0.001 \\
\hline Others & $2.866(2.291,3.584)$ & $<0.001$ & $1.228(0.970,1.555)$ & 0.088 \\
\hline LVI (presence vs. absence) & $3.477(3.000,4.029)$ & $<0.001$ & $1.254(1.071,1.468)$ & 0.005 \\
\hline VPI (presence vs. absence) & $2.380(2.075,2.730)$ & $<0.001$ & $1.478(1.281,1.704)$ & $<0.001$ \\
\hline Adjuvant chemotherapy (yes vs. no) & $4.179(3.666,4.763)$ & $<0.001$ & $0.927(0.770,1.116)$ & 0.425 \\
\hline Adjuvant radiotherapy (yes vs. no) & $4.841(3.959,5.920)$ & $<0.001$ & $1.583(1.280,1.959)$ & $<0.001$ \\
\hline
\end{tabular}

RFS, recurrence-free survival; HR, hazard ratio; Cl, Confidence interval; GGO, ground-glass opacity; SIl, systemic immune-inflammation index; MPA, micropapillary pattern-predominant adenocarcinoma; SPA, solid pattern-predominant adenocarcinoma; SQCC, squamous cell carcinoma; LVI, lymphovascular invasion; VPI, visceral pleural invasion.

Survival analyses showed higher SII indicated worse OS $(\mathrm{P}<0.0001$, Figure $1 B)$. Univariate Cox proportional hazard regressions revealed that age $(\mathrm{P}<0.001)$, sex $(\mathrm{P}<0.001)$, smoking history $(\mathrm{P}<0.001)$, GGO components $(\mathrm{P}<0.001)$, SII $(\mathrm{P}<0.001)$, p-TNM stage $(\mathrm{P}<0.001)$, histology $(\mathrm{P}<0.001)$, LVI $(\mathrm{P}<0.001)$, VPI $(\mathrm{P}<0.001)$, adjuvant chemotherapy $(\mathrm{P}<0.001)$, and adjuvant radiotherapy $(\mathrm{P}<0.001)$ were associated with impaired OS (Table S2). The multivariate Cox hazard regressions revealed age (HR: $1.029,95 \%$ CI: 1.018-1.039, $\mathrm{P}<0.001$ ), GGO components (HR: 4.111, $95 \%$ CI: 2.685-6.294, $\mathrm{P}<0.001$ ), SII (HR: 1.227, 95\% CI: $1.018-$ 1.480, $\mathrm{P}=0.032), \mathrm{p}-\mathrm{TNM}$ stage $(0 / \mathrm{I}$, reference; II, HR: 2.334, 95\% CI: 1.719-3.170, P<0.001; III, HR: 5.644, 95\% CI: 4.280-7.444, $\mathrm{P}<0.001$; overall $\mathrm{P}<0.001$ ), histology (nonMPA/SPA adenocarcinoma, reference; MPA/SPA, HR: 1.309, 95\% CI: 1.002-1.708, P=0.048; SQCC, HR: 1.097,
95\% CI: 0.856-1.406, $\mathrm{P}=0.462$; others, HR: $1.627,95 \% \mathrm{CI}$ : 1.195-2.216, $\mathrm{P}=0.002$; overall $\mathrm{P}=0.008$ ), VPI (HR: 1.512, 95\% CI: $1.240-1.842, \mathrm{P}<0.001)$, adjuvant chemotherapy (HR: 0.685 , 95\% CI: 0.536-0.875), $\mathrm{P}=0.002$ ), adjuvant radiotherapy (HR: 1.389, 95\% CI: $1.038-1.860, \mathrm{P}=0.027$ ) indicated worse OS independently (Table S2).

\section{Subgroup analysis}

To fully investigate the prognostic value of SII, HRs of SII in sub-group patients using RFS were calculated (Table 3). SII had prognostic value in patients with different age $(\leq 60$, HR: $1.617,95 \%$ CI: 1.348-1.941, $\mathrm{P}<0.001$; >60, HR: 1.620 , 95\% CI: 1.357-1.932, $\mathrm{P}<0.001$ ), sex (male, HR: 1.397, $95 \%$ CI: $1.195-1.633, \mathrm{P}<0.001$; female, HR: $1.746,95 \% \mathrm{CI}$ : 1.401-2.177, $\mathrm{P}<0.001$ ), smoking history (never, HR: 1.648, 
Table 3 HRs of SII on RFS stratified by different clinicopathological factors $(n=3,984)$

\begin{tabular}{|c|c|c|c|c|}
\hline \multirow{2}{*}{ Variables } & \multirow{2}{*}{$\mathrm{HR}$} & \multicolumn{2}{|c|}{$95 \% \mathrm{Cl}$} & \multirow{2}{*}{$P$ value } \\
\hline & & Lower & Upper & \\
\hline \multicolumn{5}{|l|}{ Age, years } \\
\hline$\leq 60$ & 1.617 & 1.348 & 1.941 & $<0.001$ \\
\hline$>60$ & 1.620 & 1.357 & 1.932 & $<0.001$ \\
\hline \multicolumn{5}{|l|}{ Sex } \\
\hline Male & 1.397 & 1.195 & 1.633 & $<0.001$ \\
\hline Female & 1.746 & 1.401 & 2.177 & $<0.001$ \\
\hline \multicolumn{5}{|l|}{ Smoking history } \\
\hline Never & 1.648 & 1.376 & 1.974 & $<0.001$ \\
\hline Ever & 1.379 & 1.150 & 1.654 & 0.001 \\
\hline \multicolumn{5}{|l|}{ p-TNM stage } \\
\hline $0 / 1$ & 1.603 & 1.288 & 1.994 & $<0.001$ \\
\hline $\mathrm{II} / \mathrm{III}$ & 0.987 & 0.844 & 1.154 & 0.869 \\
\hline \multicolumn{5}{|l|}{ GGO components } \\
\hline Absence & 1.238 & 1.084 & 1.415 & 0.002 \\
\hline Presence & 1.257 & 0.801 & 1.971 & 0.320 \\
\hline \multicolumn{5}{|l|}{ Histology } \\
\hline Adenocarcinoma & 1.678 & 1.435 & 1.962 & $<0.001$ \\
\hline Non-adenocarcinoma & 1.181 & 0.943 & 1.480 & 0.148 \\
\hline \multicolumn{5}{|l|}{ LVI } \\
\hline Absence & 1.670 & 1.442 & 1.933 & $<0.001$ \\
\hline Presence & 1.207 & 0.936 & 1.556 & 0.148 \\
\hline \multicolumn{5}{|l|}{ VPI } \\
\hline Absence & 1.656 & 1.422 & 1.929 & $<0.001$ \\
\hline Presence & 1.497 & 1.192 & 1.881 & 0.001 \\
\hline
\end{tabular}

HR, hazard ratio; SII, systemic immune-inflammation index; RFS, recurrence-free survival; $\mathrm{Cl}$, confidence interval; GGO, groundglass opacity; LVI, lymphovascular invasion; VPI, visceral pleural invasion.

95\% CI: 1.376-1.974, $\mathrm{P}<0.001$; ever, HR: $1.379,95 \% \mathrm{CI}$ : 1.150-1.654, $\mathrm{P}=0.001$ ), or VPI status (absence, HR: 1.656, 95\% CI: 1.422-1.929, $\mathrm{P}<0.001$; presence, HR: 1.497, 95\% CI: $1.192-1.881, \mathrm{P}=0.001)$. However, the prognostic value of SII varied with the patients with different $\mathrm{p}$-TNM stage (0/I, HR: 1.603, 95\% CI: 1.288-1.994, P<0.001; II/III, HR: 0.987, 95\% CI: 0.844-1.154, P=0.869), GGO components (absence, HR: 1.238, 95\% CI: 1.084-1.415, P=0.002; presence, HR: 1.257, 95\% CI: 0.801-1.971, $\mathrm{P}=0.320)$, and histology (adenocarcinoma, HR: 1.678, 95\% CI: $1.435-$ 1.962, $\mathrm{P}<0.001$; non-adenocarcinoma, HR: $1.181,95 \%$ CI: $0.943-1.480, \mathrm{P}=0.148)$. To further validate the results, we conducted the same analyses in patients after multiple imputation for missing value $(n=4,220)$. The results were also consistent (Table S3).

Kaplan-Meier analyses were used to further investigate the prognostic value of SII. SII was an adverse predictor of RFS in NSCLC patients with stage $0 / \mathrm{I}$ disease $(\mathrm{P}<0.001$, Figure $2 A$ ), while it was not in those with stage II/III disease $(\mathrm{P}=0.87$, Figure $2 B)$. There was prognostic value of $\mathrm{SII}$ in NSCLC patients with solid nodules $(\mathrm{P}=0.002$, Figure $3 A)$, while there was not in those with pure or mixed GGO $(\mathrm{P}=0.32$, Figure $3 B)$. SII was able to predict RFS in patients with lung adenocarcinoma $(\mathrm{P}<0.001$, Figure $4 A)$, while it was not in those with non-adenocarcinoma $(\mathrm{P}=0.15$, Figure $4 B)$. Competing risk analyses also confirmed the distinct prognostic value in patients with different stage, GGO appearance, and histology. The results were also consistent with previous conclusions (Figure S3).

\section{Discussion}

Inflammation has been identified as a key factor in pathogenesis, tumor progression, and metastasis of malignancies $(2,4)$. SII, as an inflammatory marker, is able to predict survival outcomes in a number of solid tumors, including NSCLC (5-8,14-16). In this large-scale study, we revealed that elevated SII independently correlated with poor RFS and OS of patients with surgically-resected NSCLC. In addition, we investigated the prognostic value of SII in sub-group analyses and found SII was a stagedependent prognostic factor. SII was able to predict RFS solely in patients with stage I disease rather than stage II/III. Furthermore, the prognostic role was observed solely in solid nodules or lung adenocarcinoma. To our knowledge, this is the first study to comprehensively investigate the prognostic value of SII together with TNM stage, radiological appearance, and histology. This study helps us select patients, who might benefit from the clinical use of SII.

Increasing studies have demonstrated that SII was a significant prognostic factor in patients with NSCLC $(5,7,14,15)$. Wang et al. (7) conducted a meta-analysis regarding of the prognostic value of pretreatment SII in patients with NSCLC. After evaluating nine studies 

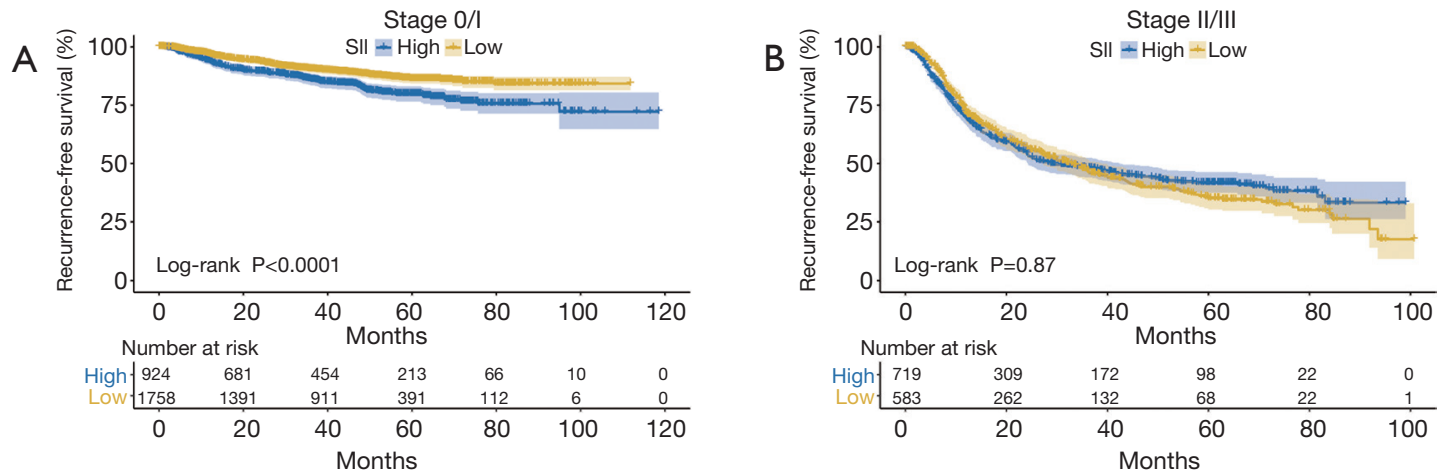

Figure 2 Kaplan-Meier curves of RFS according to the level of SII in patients with stage I (A) and stage II/III (B) disease. High SII was defined as more than 479, while low SII as no more than 479. RFS, recurrence-free survival; SII, systemic immune-inflammation index.
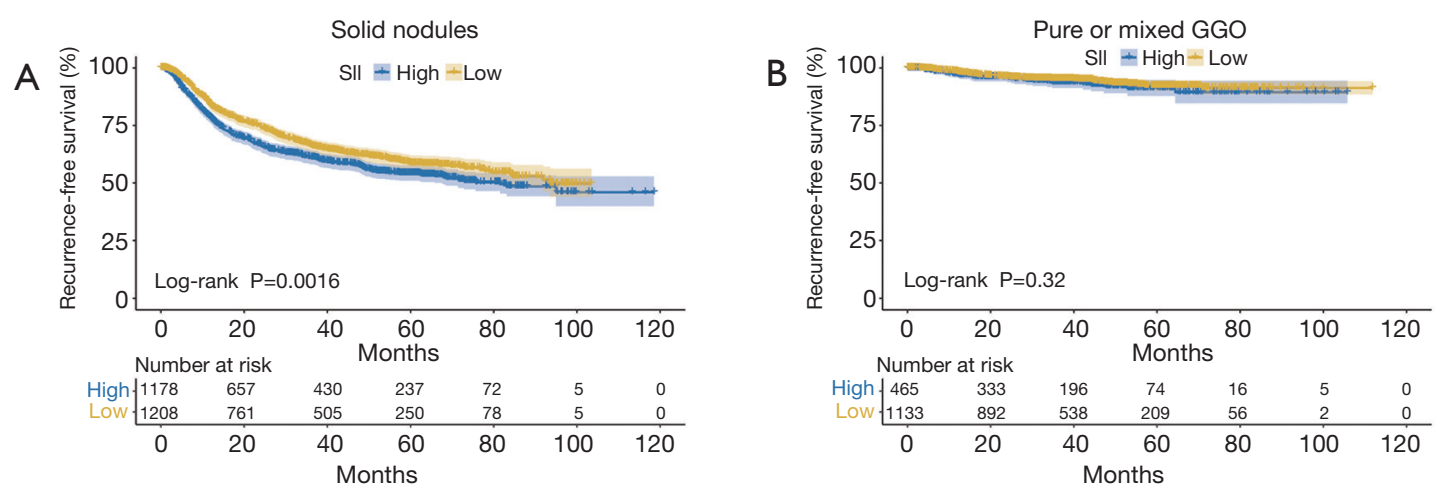

Figure 3 Kaplan-Meier curves of RFS according to the level of SII in patients with solid nodules (A) and pure or mixed GGO (B). High SII was defined as more than 479, while low SII as no more than 479. RFS, recurrence-free survival; SII, systemic immune-inflammation index; GGO, ground-glass opacity.
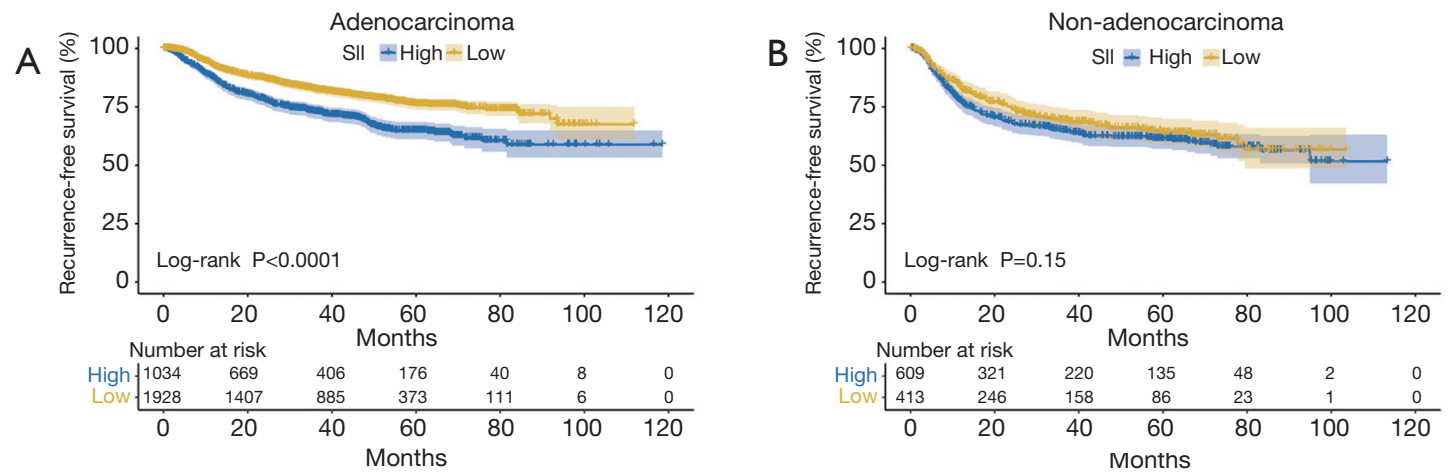

Figure 4 Kaplan-Meier curves of RFS according to the level of SII in patients with adenocarcinoma (A) and none adenocarcinoma (B). High SII was defined as more than 479, while low SII as no more than 479. RFS, recurrence-free survival; SII, systemic immune-inflammation index. 
involving 2,441 patients, they found that the pretreatment SII served as a useful prognostic indicator and showed a much higher prognostic value than neutrophil to lymphocyte ratio (NLR) or the platelet to lymphocyte ratio (PLR). In this study, based on a large-scale population containing 3,984 patients, we confirmed the prognostic value of SII in surgically-resected NSCLC. Since blood test results were inexpensive and often routinely performed, SII was a non-invasive and easily accessible candidate for the prognostic factor in NSCLC.

The prognostic value of SII in patients with different characteristics lacks evidence, and the results are crucial for the clinical utilization of SII in the correct population. In this study, we found that the prognostic value of SII was observed only in patients with stage I NSCLC, rather than those with stage II/III NSCLC. The possible reason might be that immune cells tended to be non-functional in stage II/III NSCLC. Therefore, SII seemed to lack of prognostic role in these patients. In addition, an increasing number of studies revealed GGO was a favorable factor for the survival of lung cancer $(9,11,17)$. In this study, the prognostic value of SII was present solely in patients with solid nodules, while it was not in those with pure or mixed GGO. The excellent survival of GGO might lead to the results. Furthermore, the prognostic value of SII was shown solely in patients with adenocarcinoma, rather than nonadenocarcinoma patients. The patients with SQCC are prone to develop pneumonia, and it might affect the results of blood test. As a result, when SII is used clinically, the selective criteria of patients should be carefully considered.

SII was generated from the absolute count of platelet, neutrophil, and lymphocyte. Ji et al. (18) reviewed 234 stage I NSCLC patients and found out that evaluated platelet count was a strong predictor of poor prognosis. Platelet was able to secrete a number of $\alpha$-granules, which was associated with proliferation and angiogenesis of tumor (19). As for neutrophil, the count was also reported to be an independent prognostic factor in patients with NSCLC (20). Zhang et al. (21) investigated 142 patients with NSCLC and found preoperative lymphocyte count was a favorable prognostic factor. In conclusion, the counts of platelet, neutrophil, and lymphocyte were able to predict outcomes of NSCLC separately, resulting in the fact that the parameter based on three variables (SII) exhibited higher prognostic value than NLR or PLR.

Immunotherapy has been reported to significantly prolong the survival of patients with malignancies (22-24).
The reliable biomarkers for immunotherapy are important for select patients with potential survival benefits. Inflammatory indexes, such as NLR, have were able to predict response to immunotherapy (25). In NSCLC, Diem et al. (26) demonstrated that NLR and PLR were prognostic markers in patients treated with nivolumab. In 2019, SII was also reported to be prognostic in immunotherapytreated patients (27). Although there are limited data regarding this issue, SII seems to be a promising biomarker for immunotherapy in lung cancer.

However, there are some limitations of this study. Firstly, due to the retrospective nature of the study, bias inevitably exits in patient enrollment. Secondly, SII can be affected by concurrent infection and drug intake. However, thanks to the large population, the effects of concurrent infection and drug intake could be minimized. Thirdly, it is a singleinstitution study, and future multi-center study is urged.

\section{Conclusions}

In summary, this study investigated the prognostic value of SII comprehensively in operable NSCLC. High SII was associated with worse survival independently, and its prognostic role was exhibited solely in NSCLC patients with stage I disease, solid nodules, and adenocarcinoma. This study helped specify the target population for clinical use of SII.

\section{Acknowledgments}

Funding: This work was supported by the National Natural Science Foundation of China (81930073 and 81772466), Shanghai Science and Technology Innovation Action Project (20JC1417200), Shanghai Municipal Science and Technology Major Project (2017SHZDZX01, VBH1323001/026), Shanghai Municipal Key Clinical Specialty Project (SHSLCZDZK02104), Pilot Project of Fudan University (IDF159045), and Shanghai Sailing Program (19YF1408800).

\section{Footnote}

Reporting Checklist: The authors have completed the REMARK reporting checklist. Available at https://dx.doi. org/10.21037/tlcr-21-267

Data Sharing Statement: Available at https://dx.doi. 
org/10.21037/tlcr-21-267

Peer Review File: Available at https://dx.doi.org/10.21037/ tlcr-21-267

Conflicts of Interest: All authors have completed the ICMJE uniform disclosure form (available at https://dx.doi. org/10.21037/tlcr-21-267). The authors have no conflicts of interest to declare.

Ethical Statement: The authors are accountable for all aspects of the work in ensuring that questions related to the accuracy or integrity of any part of the work are appropriately investigated and resolved. The study was conducted in accordance with the Declaration of Helsinki (as revised in 2013). The study was approved by Institutional Review Board of FUSCC (IRB\#090977-1) and individual consent for this retrospective analysis was waived.

Open Access Statement: This is an Open Access article distributed in accordance with the Creative Commons Attribution-NonCommercial-NoDerivs 4.0 International License (CC BY-NC-ND 4.0), which permits the noncommercial replication and distribution of the article with the strict proviso that no changes or edits are made and the original work is properly cited (including links to both the formal publication through the relevant DOI and the license). See: https://creativecommons.org/licenses/by-nc-nd/4.0/.

\section{References}

1. Iwasaki A, Medzhitov R. Regulation of adaptive immunity by the innate immune system. Science 2010;327:291-5.

2. Mantovani A, Allavena P, Sica A, et al. Cancer-related inflammation. Nature 2008;454:436-44.

3. Yang L, Karin M. Roles of tumor suppressors in regulating tumor-associated inflammation. Cell Death Differ 2014;21:1677-86.

4. McMillan DC. Cancer and systemic inflammation: stage the tumour and stage the host. Br J Cancer 2013;109:529.

5. Li X, Hu P, Liu J, et al. Systemic immune-inflammation index predicted overall survival and radiosensitivity in advanced non-small-cell lung cancer. Future Oncol 2020;16:103-15

6. Tong YS, Tan J, Zhou XL, et al. Systemic immuneinflammation index predicting chemoradiation resistance and poor outcome in patients with stage III non-small cell lung cancer. J Transl Med 2017;15:221.
7. Wang Y, Li Y, Chen P, et al. Prognostic value of the pretreatment systemic immune-inflammation index (SII) in patients with non-small cell lung cancer: a meta-analysis. Ann Transl Med 2019;7:433.

8. Hong X, Cui B, Wang M, et al. Systemic immuneinflammation index, based on platelet counts and neutrophil-lymphocyte ratio, is useful for predicting prognosis in small cell lung cancer. Tohoku J Exp Med 2015;236:297-304.

9. Fu F, Zhang Y, Wen Z, et al. Distinct prognostic factors in patients with stage I non-small cell lung cancer with radiologic part-solid or solid lesions. J Thorac Oncol 2019;14:2133-42.

10. Hansell DM, Bankier AA, MacMahon H, et al. Fleischner Society: glossary of terms for thoracic imaging. Radiology 2008;246:697-722.

11. Ye T, Deng L, Wang S, et al. Lung adenocarcinomas manifesting as radiological part-solid nodules define a special clinical subtype. J Thorac Oncol 2019;14:617-27.

12. Travis WD, Brambilla E, Nicholson AG, et al. The 2015 World Health Organization Classification of Lung Tumors: Impact of Genetic, Clinical and Radiologic Advances Since the 2004 Classification. J Thorac Oncol 2015;10:1243-60.

13. Goldstraw P, Chansky K, Crowley J, et al. The IASLC Lung Cancer Staging Project: Proposals for Revision of the TNM Stage Groupings in the Forthcoming (Eighth) Edition of the TNM Classification for Lung Cancer. J Thorac Oncol 2016;11:39-51.

14. Berardi R, Santoni M, Rinaldi S, et al. Pre-treatment systemic immune-inflammation represents a prognostic factor in patients with advanced non-small cell lung cancer. Ann Transl Med 2019;7:572.

15. Guo W, Cai S, Zhang F, et al. Systemic immuneinflammation index (SII) is useful to predict survival outcomes in patients with surgically resected non-small cell lung cancer. Thorac Cancer 2019;10:761-8.

16. Jomrich G, Paireder M, Kristo I, et al. High systemic immune-inflammation index is an adverse prognostic factor for patients with gastroesophageal adenocarcinoma. Ann Surg 2021;273:532-41.

17. Hattori A, Hirayama S, Matsunaga T, et al. Distinct clinicopathologic characteristics and prognosis based on the presence of ground glass opacity component in clinical stage IA lung adenocarcinoma. J Thorac Oncol 2019;14:265-75.

18. Ji Y, Sheng L, Du X, et al. Elevated platelet count is a strong predictor of poor prognosis in stage I non-small cell 
lung cancer patients. Platelets 2015;26:138-42.

19. Alvarez RH, Kantarjian HM, Cortes JE. Biology of platelet-derived growth factor and its involvement in disease. Mayo Clin Proc 2006;81:1241-57.

20. Teramukai S, Kitano T, Kishida Y, et al. Pretreatment neutrophil count as an independent prognostic factor in advanced non-small-cell lung cancer: an analysis of Japan Multinational Trial Organisation LC00-03. Eur J Cancer 2009;45:1950-8.

21. Zhang J, Huang SH, Li H, et al. Preoperative lymphocyte count is a favorable prognostic factor of disease-free survival in non-small-cell lung cancer. Med Oncol 2013;30:352.

22. Nghiem PT, Bhatia S, Lipson EJ, et al. PD-1 Blockade with pembrolizumab in advanced Merkel-cell carcinoma. N Engl J Med 2016;374:2542-52.

23. Ansell SM, Lesokhin AM, Borrello I, et al. PD-1 blockade with nivolumab in relapsed or refractory Hodgkin's

Cite this article as: Fu F, Deng C, Wen Z, Gao Z, Zhao Y, Han H, Zheng S, Wang S, Li Y, Hu H, Zhang Y, Chen H. Systemic immune-inflammation index is a stage-dependent prognostic factor in patients with operable non-small cell lung cancer. Transl Lung Cancer Res 2021;10(7):3144-3154. doi: 10.21037/tlcr-21-267 lymphoma. N Engl J Med 2015;372:311-9.

24. Hodi FS, O'Day SJ, McDermott DF, et al. Improved survival with ipilimumab in patients with metastatic melanoma. N Engl J Med 2010;363:711-23.

25. Kuzman JA, Stenehjem DD, Merriman J, et al. Neutrophil-lymphocyte ratio as a predictive biomarker for response to high dose interleukin-2 in patients with renal cell carcinoma. BMC Urol 2017;17:1.

26. Diem S, Schmid S, Krapf M, et al. Neutrophil-toLymphocyte ratio (NLR) and Platelet-to-Lymphocyte ratio (PLR) as prognostic markers in patients with nonsmall cell lung cancer (NSCLC) treated with nivolumab. Lung Cancer 2017;111:176-81.

27. Liu J, Li S, Zhang S, et al. Systemic immune-inflammation index, neutrophil-to-lymphocyte ratio, platelet-tolymphocyte ratio can predict clinical outcomes in patients with metastatic non-small-cell lung cancer treated with nivolumab. J Clin Lab Anal 2019;33:e22964. 

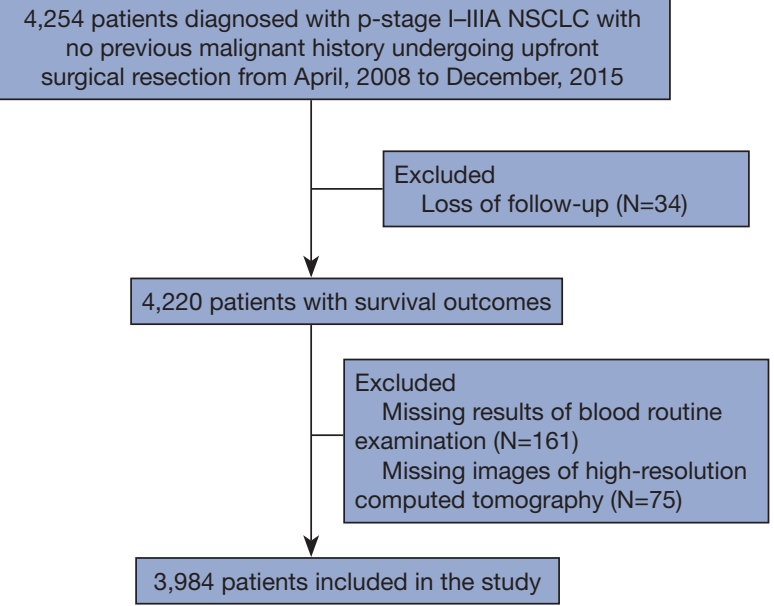

Figure S1 The workflow of this study. NSCLC, non-small cell lung cancer.
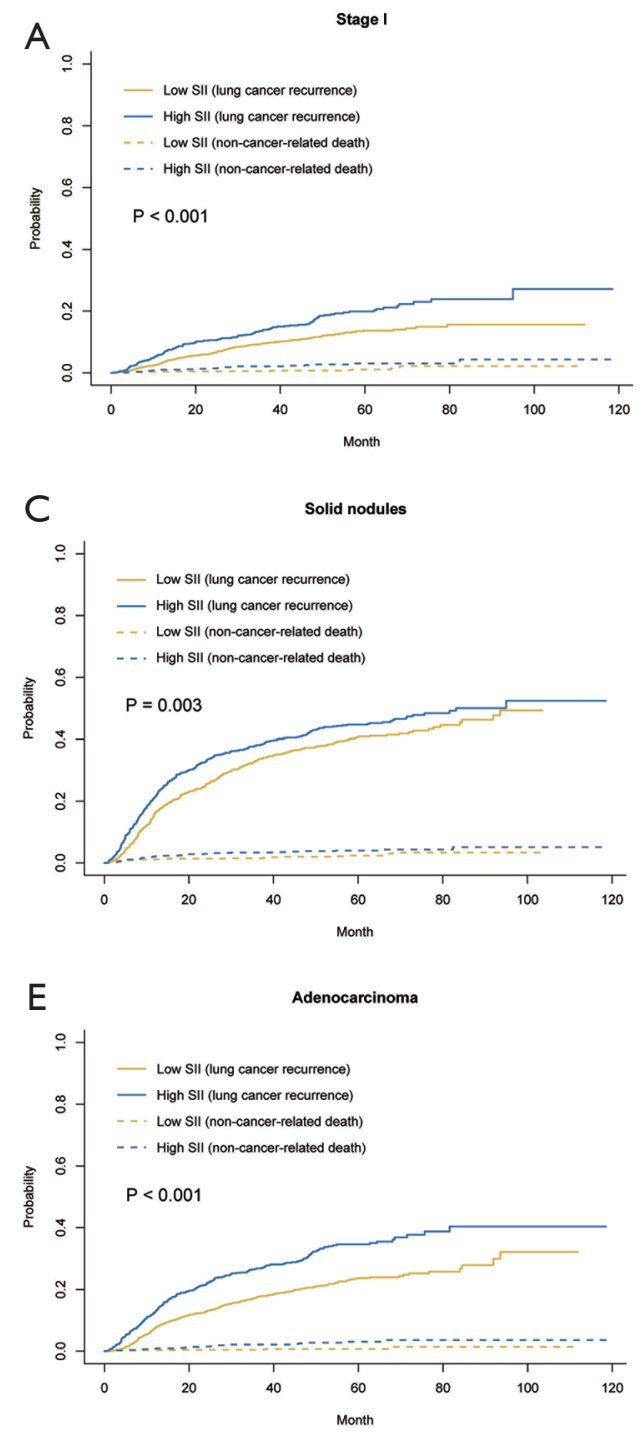

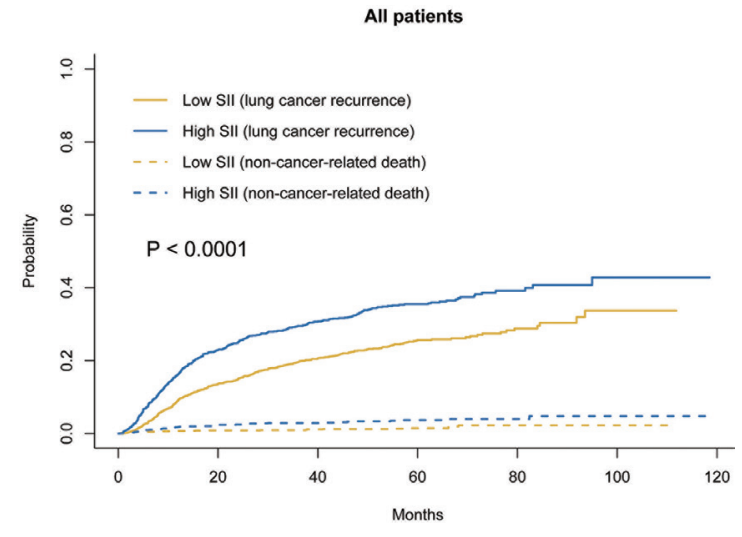

Figure S2 Cumulative recurrence rate according to the level of SII using competing risk analyses. High SII was defined as more than 479, while low SII as no more than 479. SII, systemic immuneinflammation index.
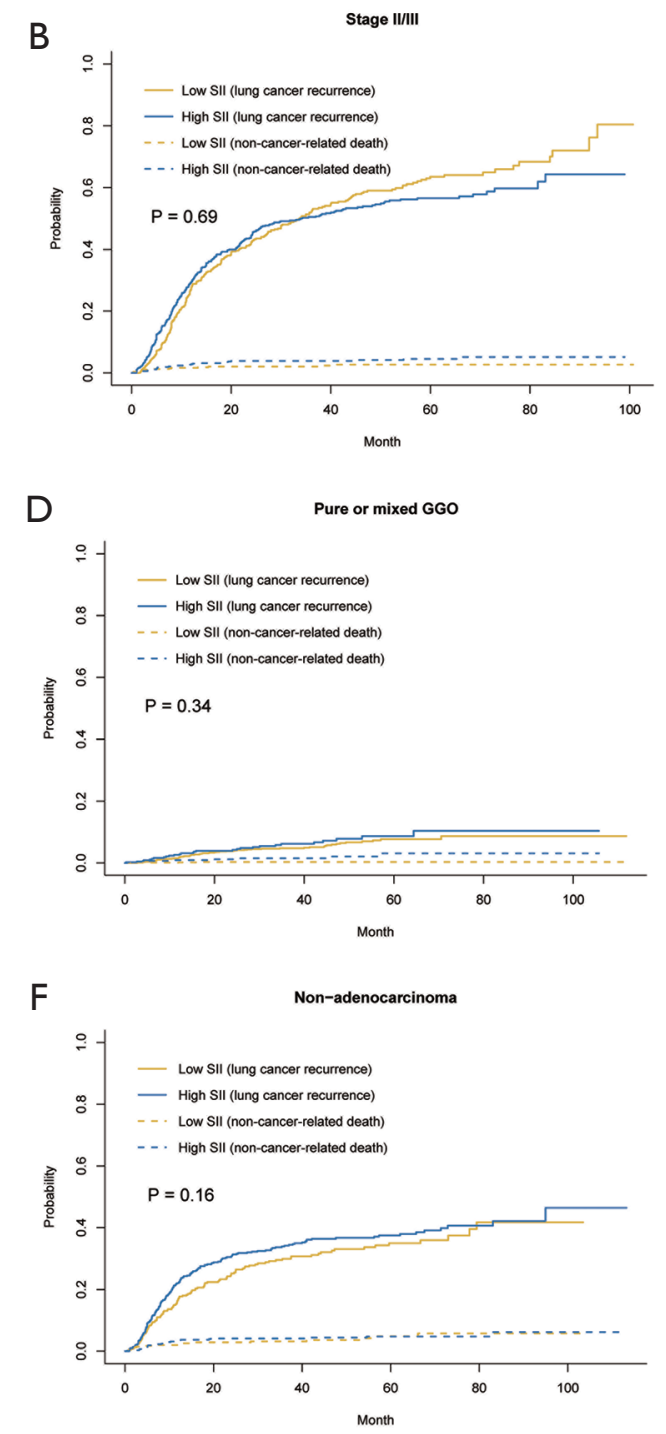

Figure S3 Cumulative recurrence rate according to the level of SII using competing risk analyses in patients with stage I disease (A), stage II/III disease (B), solid nodules (C), pure or mixed GGO (D), adenocarcinoma (E), or none adenocarcinoma (F). High SII was defined as more than 479, while low SII as no more than 479. SII, systemic immune-inflammation index; GGO, ground-glass opacity. 
Table S1 Univariate and multivariable Cox regression analysis of factors associated with RFS after multiple imputation ( $\mathrm{n}=4,220)$

\begin{tabular}{|c|c|c|c|c|}
\hline \multirow{2}{*}{ Variables } & \multicolumn{2}{|c|}{ Univariate } & \multicolumn{2}{|c|}{ Multivariate } \\
\hline & $\mathrm{HR}(95 \% \mathrm{Cl})$ & $\mathrm{P}$ & $\mathrm{HR}(95 \% \mathrm{Cl})$ & $P$ \\
\hline Age & $1.014(1.008,1.021)$ & $<0.001$ & $1.009(1.002,1.016)$ & 0.012 \\
\hline Sex (male vs. female) & $1.821(1.600,2.074)$ & $<0.001$ & $1.196(1.002,1.427)$ & 0.047 \\
\hline Smoking history & $1.677(1.483,1.897)$ & $<0.001$ & $1.119(0.946,1.324)$ & 0.190 \\
\hline GGO components (absence vs. presence) & $6.916(5.651,8.465)$ & $<0.001$ & $3.139(2.491,3.955)$ & $<0.001$ \\
\hline SIl (high vs. low) & $1.647(1.457,1.863)$ & $<0.001$ & $1.151(1.012,1.309)$ & 0.032 \\
\hline p-TNM stage & & $<0.001$ & & $<0.001$ \\
\hline $0 / 1$ & Reference & & Reference & \\
\hline II & $3.812(3.207,4.531)$ & $<0.001$ & $2.308(1.870,2.850)$ & $<0.001$ \\
\hline III & $8.444(7.335,9.720)$ & $<0.001$ & $4.527(3.710,5.523)$ & $<0.001$ \\
\hline Histology & & $<0.001$ & & $<0.001$ \\
\hline Non-MPA/SPA adenocarcinoma & Reference & & Reference & \\
\hline MPA/SPA & $3.165(2.663,3.761)$ & $<0.001$ & $1.211(1.012,1.450)$ & 0.037 \\
\hline SQCC & $1.832(1.573,2.134)$ & $<0.001$ & $0.746(0.625,0.890)$ & 0.001 \\
\hline Others & $2.799(2.245,3.489)$ & $<0.001$ & $1.221(0.968,1.540)$ & 0.092 \\
\hline LVI (presence vs. absence) & $3.521(3.052,4.062)$ & $<0.001$ & $1.284(1.102,1.497)$ & 0.001 \\
\hline VPI (presence vs. absence) & $2.410(2.110,2.753)$ & $<0.001$ & $1.502(1.308,1.725)$ & $<0.001$ \\
\hline Adjuvant chemotherapy (yes vs. no) & $4.127(3.636,4.684)$ & $<0.001$ & $0.912(0.763,1.090)$ & 0.312 \\
\hline Adjuvant radiotherapy (yes vs. no) & $4.799(3.945,5.837)$ & $<0.001$ & $1.627(1.323,2.002)$ & $<0.001$ \\
\hline
\end{tabular}

RFS, recurrence-free survival; HR, hazard ratio; Cl, Confidence interval; GGO, ground-glass opacity; SII, systemic immune-inflammation index; MPA, micropapillary pattern-predominant adenocarcinoma; SPA, solid pattern-predominant adenocarcinoma; SQCC, squamous cell carcinoma; LVI, Iymphovascular invasion; VPI, visceral pleural invasion.

Table S2 Univariate and multivariable Cox regression analysis of factors associated with OS $(\mathrm{n}=3,984)$

\begin{tabular}{|c|c|c|c|c|}
\hline \multirow{2}{*}{ Variables } & \multicolumn{2}{|l|}{ Univariate } & \multicolumn{2}{|c|}{ Multivariate } \\
\hline & $\mathrm{HR}(95 \% \mathrm{Cl})$ & $P$ & $\mathrm{HR}(95 \% \mathrm{Cl})$ & $P$ \\
\hline Age & $1.035(1.025,1.045)$ & $<0.001$ & $1.029(1.018,1.039)$ & $<0.001$ \\
\hline Sex (male vs. female) & $2.156(1.772,2.623)$ & $<0.001$ & $1.275(0.979,1.661)$ & 0.071 \\
\hline Smoking history & $1.929(1.613,2.307)$ & $<0.001$ & $1.144(0.898,1.457)$ & 0.278 \\
\hline GGO components (absence vs. presence) & $11.185(7.584,16.494)$ & $<0.001$ & $4.111(2.685,6.294)$ & $<0.001$ \\
\hline SIl (high vs. low) & $1.841(1.540,2.202)$ & $<0.001$ & $1.227(1.018,1.480)$ & 0.032 \\
\hline p-TNM stage & & $<0.001$ & & $<0.001$ \\
\hline $0 / 1$ & Reference & & Reference & \\
\hline II & $3.672(2.819,4.782)$ & $<0.001$ & $2.334(1.719,3.170)$ & $<0.001$ \\
\hline III & $8.344(6.789,10.255)$ & $<0.001$ & $5.644(4.280,7.444)$ & $<0.001$ \\
\hline Histology & & $<0.001$ & & 0.008 \\
\hline Non-MPA/SPA adenocarcinoma & Reference & & Reference & \\
\hline MPA/SPA & $3.152(2.433,4.083)$ & $<0.001$ & $1.309(1.002,1.708)$ & 0.048 \\
\hline SQCC & $2.626(2.120,3.252)$ & $<0.001$ & $1.097(0.856,1.406)$ & 0.462 \\
\hline Others & $3.854(2.881,5.156)$ & $<0.001$ & $1.627(1.195,2.216)$ & 0.002 \\
\hline LVI (presence vs. absence) & $2.884(2.344,3.548)$ & $<0.001$ & $1.083(0.868,1.351)$ & 0.480 \\
\hline VPI (presence vs. absence) & $2.236(1.847,2.707)$ & $<0.001$ & $1.512(1.240,1.842)$ & $<0.001$ \\
\hline Adjuvant chemotherapy (yes vs. no) & $3.297(2.747,3.956)$ & $<0.001$ & $0.685(0.536,0.875)$ & 0.002 \\
\hline Adjuvant radiotherapy (yes vs. no) & $3.826(2.901,5.046)$ & $<0.001$ & $1.389(1.038,1.860)$ & 0.027 \\
\hline
\end{tabular}

OS, overall survival; HR, hazard ratio; $\mathrm{Cl}$, confidence interval; GGO, ground-glass opacity; SII, systemic immune-inflammation index; MPA, micropapillary pattern-predominant adenocarcinoma; SPA, solid pattern-predominant adenocarcinoma; SQCC, squamous cell carcinoma; LVI, lymphovascular invasion; VPI, visceral pleural invasion. 
Table S3 HRs of SII on RFS stratified by different clinicopathological factors after multiple imputation $(n=4,220)$

\begin{tabular}{|c|c|c|c|c|}
\hline \multirow{2}{*}{ Variables } & \multirow{2}{*}{$\mathrm{HR}$} & \multicolumn{2}{|c|}{$95 \% \mathrm{Cl}$} & \multirow{2}{*}{$P$ value } \\
\hline & & Lower & Upper & \\
\hline \multicolumn{5}{|l|}{ Age } \\
\hline$\leq 60$ & 1.651 & 1.385 & 1.969 & $<0.001$ \\
\hline$>60$ & 1.622 & 1.366 & 1.927 & $<0.001$ \\
\hline \multicolumn{5}{|l|}{ Sex } \\
\hline Male & 1.405 & 1.207 & 1.635 & $<0.001$ \\
\hline Female & 1.770 & 1.430 & 2.190 & $<0.001$ \\
\hline \multicolumn{5}{|l|}{ Smoking history } \\
\hline Never & 1.653 & 1.388 & 1.969 & $<0.001$ \\
\hline Ever & 1.401 & 1.175 & 1.672 & $<0.001$ \\
\hline \multicolumn{5}{|l|}{ p-TNM stage } \\
\hline $0 / 1$ & 1.565 & 1.266 & 1.934 & $<0.001$ \\
\hline II/III & 0.995 & 0.854 & 1.158 & 0.947 \\
\hline \multicolumn{5}{|l|}{ GGO components } \\
\hline Absence & 1.237 & 1.086 & 1.408 & 0.001 \\
\hline Presence & 1.386 & 0.926 & 2.073 & 0.113 \\
\hline \multicolumn{5}{|l|}{ Histology } \\
\hline Adenocarcinoma & 1.665 & 1.431 & 1.937 & $<0.001$ \\
\hline Non-adenocarcinoma & 1.243 & 0.997 & 1.550 & 0.054 \\
\hline \multicolumn{5}{|l|}{ LVI } \\
\hline Absence & 1.677 & 1.455 & 1.933 & $<0.001$ \\
\hline Presence & 1.270 & 0.992 & 1.625 & 0.058 \\
\hline \multicolumn{5}{|l|}{ VPI } \\
\hline Absence & 1.645 & 1.419 & 1.908 & $<0.001$ \\
\hline Presence & 1.561 & 1.251 & 1.947 & $<0.001$ \\
\hline
\end{tabular}

HR, hazard ratio; SII, systemic immune-inflammation index; RFS, recurrence-free survival; Cl, confidence interval; GGO, ground-glass opacity; LVI, lymphovascular invasion; VPI, visceral pleural invasion. 